\title{
Adaptive Regulation of Time Varying Disturbances via Weighted Robust Estimation and Automatic Controller Tuning
}

\author{
Huazhen Fang ${ }^{1}$ and Raymond A. de Callafon ${ }^{2}$
}

\begin{abstract}
This paper proposes a methodology to adaptively reduce time varying and narrow band harmonic disturbances via the estimation of a feedback controller transfer function parameterized in a Youla-Kucera parametrization. The proposed adaptive feedback regulation simultaneously minimizes the variance of a performance (output) signal and a control (input) signal in real-time. Uncertainty on the plant dynamics is taken into account by including a frequency weighting on the control signal and the solution is formulated as a Recursive Least Squares minimization. The methodology is applied to a mechanical vibration control benchmark that is part of a collaborative invited session at the conference to demonstrate how the proposed adaptive feedback regulation can effectively reduce unknown harmonic disturbances with time-varying frequency contents.
\end{abstract}

\section{INTRODUCTION}

Feedback control can be effective in reducing the effect of unknown disturbances. The more information is known on the disturbance dynamics, the better the feedback control algorithm can be tuned to alleviate the effects. A good example is the internal model principle [1] or the more general form of repetitive control [2], [3] that use resonators in the feedback controller to compensate for general periodic disturbances. Feedback compensation of periodic disturbances also can be shown to be equivalent to adaptive feedforward compensation [4] under certain conditions. The requirements on maintaining closed-loop stability in feedback control requires knowledge on the plant dynamics [5], whereas fixed linear feedback control does impose limitations on the frequency range over which disturbances can be reduced.

Limitations of fixed linear feedback control are well captured by the celebrated Bode Sensitivity integral [6], [7], [8] $\int_{0}^{\infty} \ln |S(j \omega)| d \omega=-\frac{\pi}{2} \lim _{s \rightarrow \infty} s L(s)$ for a feedback connection

$$
\begin{aligned}
y & =G u+v, v=H e \\
u & =C(r-y)
\end{aligned}
$$

of a stable system $G$ and stable controller $C$ with a loop gain $L(s)=G(s) C(s)$ and a sensitivity function $S(s)=$ $(1+L(s))^{-1}$. A challenging problem arises when the spectral contents of the disturbance, modelled by $H$ in (1), changes over time. Changes in disturbance dynamics $H$ may result in amplification of the disturbance $v(t)$ in (1) due to a gain $|S(j \omega)|>1$ of the sensitivity function. Adaptive solutions for this problem have been proposed using feedforward

\footnotetext{
${ }^{1} \mathrm{H}$. Fang is a graduate student with Dept. of Mechanical and Aerospace Engineering, University of California San Diego, La Lolla, CA 92093-0411, United States of America huafangeucsd.edu

${ }^{2}$ R.A. de Callafon is with Dept. of Mechanical and Aerospace Engineering, University of California San Diego, La Lolla, CA 92093-0411, United States of America callafon@ucsd.edu
}

control [9] that rely on linearly parametrized filters with recursive estimation techniques [10], but require additional sensors for disturbance monitoring and estimation.

Adaptive regulation [11] is a viable solution to deal with varying disturbance dynamics in feedback control. Adaptive regulation has been studied for periodic disturbances [12], [4] and with Iterative Learning Control (ILC) [13], [14], [15] and the class of filtered white noise disturbances in [11]. More recently, the ideas of adaptive regulation have been extended to specific classes of periodic disturbances [16]. Earlier work has demonstrated how such algorithms can be implemented in real-time [17] to allow adaptive regulation of time varying disturbance dynamics, even in the presence of plant uncertainty. The Robust Estimation and Automatic Controller Tuning (REACT) in [17] implements adaptive regulation by recursively minimizing the variance of a single performance signal with an application in vibration control.

Minimizing the variance of a single performance signal leads to minimum variance control solutions with possible large control signals, especially for non-minimum phase systems [18]. Compared to the earlier work on REACT in [17], the adaptive feedback regulation proposed in this paper simultaneously minimizes the variance of a performance signal and a control output signal via a so-called weighted robust estimation. The resulting weighted REACT approach allows a trade-off between minimizing output variance to reduce unknown and time varying disturbances, frequency weighted against the control energy to account for model uncertainty. It is shown in this paper that the weighted controller estimation can be formulated as a Recursive Least Squares minimization problem in which the feedback controller transfer function is parametrized in a Youla-Kucera parametrization.

The methodology is applied to a vibration control benchmark to demonstrate how the proposed adaptive feedback regulation can effectively reduce unknown harmonic disturbances. Plant uncertainty is taken into account by including a frequency weighting on the control energy. Furthermore, various periodic disturbances with jumps in the frequency contents are used to study transition effects and the performance of the proposed weighted REACT algorithm during the application.

\section{ADAPTIVE REGULATION VIA RECURSIVE CONVEX OPTIMIZATION}

\section{A. Controller parametrization}

A well-know result in controller design and optimization is the Youla-Kucera parametrization [18] that allows 
the parametrization of the class of all stabilizing feedback controllers for a given dynamical system by the freedom in a stable dynamical perturbation $Q$. The parametrization can be formulated in terms of a right coprime factorization (rcf) of a model $\hat{G}$ and the rcf of an initial stabilizing feedback controller $C_{i}$.

Definition 1: Youla-Kucera Parametrization.

Let $T\left(C_{i}, \hat{G}\right)$ denote the feedback connection of a nominal model $\hat{G}$ and an initial controller $C_{i}$ with $T\left(C_{i}, \hat{G}\right) \in \mathcal{R} \mathcal{H}_{\infty}$. All controllers $C_{Q}=N_{Q} D_{Q}^{-1}$ that satisfy $T\left(C_{Q}, \hat{G}\right) \in$ $\mathcal{R} \mathcal{H}_{\infty}$ are given by the $\operatorname{rcf}$

$$
\begin{aligned}
& N_{Q}=N_{i}+D Q \\
& D_{Q}=D_{i}-N Q
\end{aligned}, \quad Q \in \mathcal{R} \mathcal{H}_{\infty}
$$

where $(N, D)$ is a ref of $\hat{G}$ and $\left(N_{i}, D_{i}\right)$ is a $\operatorname{rcf}$ of $C_{i}$. A similar definition can also be given for a left coprime factorization (lcf) $(\tilde{N}, \tilde{D})$ of $\hat{G}$. Defining $T(C, G)$ as

$$
T(C, G)=\left[\begin{array}{c}
C \\
I
\end{array}\right](I+G C)^{-1}\left[\begin{array}{ll}
G & I
\end{array}\right]
$$

then $T(C, G) \in \mathcal{R H}_{\infty}$ for internal stability is equivalent to $\Lambda^{-1} \in \mathcal{R} \mathcal{H}_{\infty}$, with $\Lambda=\tilde{D} D_{c}+\tilde{N} N_{c}$ [6]. It is a well know result that the perturbation $Q$ can be varied to find new controllers for the nominal model $\hat{G}$. As long as $Q \in \mathcal{R} \mathcal{H}_{\infty}$ stability can be maintained for the feedback of $C_{Q}$ and $\hat{G}$.

\section{B. Mixed performance and control optimization}

Next to providing a parametrization of all stabilizing controllers, the Youla-Kucera parametrization in Definition 1 provides an another advantage for controller adaptation: all closed-loop transfer functions are linear in the controller perturbation $Q$. Considering a feedback system

$$
\begin{aligned}
& y(t)=\hat{G} u(t)+H e(t) \\
& u(t)=-C_{Q} y(t)
\end{aligned}
$$

the weighted REACT approach in this paper aims at minimizing the weighted two-norm performance measure

$$
\left\|\begin{array}{c}
\gamma u_{w} \\
y
\end{array}\right\|_{2}, \quad u_{w}(t)=W(q) u(t)
$$

that measures the combined variance of the performance signal $y(t)$ and a filtered control signal $u$ given in (2). The filter $W(q)$ is a user-specified monic stable filter, whereas the scalar $\gamma$ serves as a gain weighting in the weighted two-norm performance measure of (3).

The variance of $y(t)$ and $u(t)$ is driven by the noise disturbance $v(t)=H(q) e(t)$, where $e(t)$ is a white noise signal with a variance $\nu$ and $\Phi_{v}(\omega)=\left|H\left(e^{j \omega}\right)\right|^{2} \nu$. In the adaptive regulation, the noise spectrum $\Phi_{v}(\omega)$ is unknown and possibly time varying. The Youla-Kucera parametrization in Definition 1 allows the weighted two-norm performance measure of (3) to be written in an affine form in terms of controller perturbation $Q$.

Corollary 1: Consider a nominal model $\hat{G}$ and an initial controller $C_{i}$ with $T\left(C_{i}, \hat{G}\right) \in \mathcal{R} \mathcal{H}_{\infty}$. A controller $C_{Q}$ given in Definition 1 that minimizes (3) can be computed by

$$
\min _{Q \in \mathcal{R} \mathcal{H} \infty}\left\|W_{1} M_{22} W_{2}+W_{1} M_{21} Q M_{12} W_{2}\right\|_{2}
$$

where

$$
\begin{aligned}
M_{22} & =\left[\begin{array}{c}
N_{i} \\
D_{i}
\end{array}\right] \Lambda_{i}^{-1}\left[\begin{array}{ll}
\tilde{N} & \tilde{D}
\end{array}\right]=T\left(C_{i}, \hat{G}\right) \\
M_{21} & =\left[\begin{array}{c}
0 \\
-N
\end{array}\right] \\
M_{12} & =\Lambda_{i}^{-1}\left[\begin{array}{c}
\tilde{N} \\
\tilde{D}
\end{array}\right]=D_{i}^{-1}\left(I+C_{i} \hat{G}\right)^{-1}\left[\begin{array}{ll}
\hat{G} & I
\end{array}\right] \\
W_{1} & =\left[\begin{array}{cc}
-\gamma W & 0 \\
0 & I
\end{array}\right], W_{2}=\left[\begin{array}{c}
0 \\
H
\end{array}\right]
\end{aligned}
$$

and $\Lambda_{i}=\tilde{D} D_{i}+\tilde{N} N_{i} \in \mathcal{R} \mathcal{H}_{\infty}$.

The proof of Corollary 1 is straightforward by recognizing that $\Lambda_{i}=\tilde{D} D_{i}+\tilde{N} N_{i} \in \mathcal{R} \mathcal{H}_{\infty}$ and

$$
\begin{aligned}
& T\left(C_{Q}, \hat{G}\right)=\left[\begin{array}{l}
N_{i}+D Q \\
D_{i}-N Q
\end{array}\right] \Lambda_{i}^{-1}\left[\begin{array}{ll}
\tilde{N} & \tilde{D}
\end{array}\right] \\
& =\left[\begin{array}{l}
N_{i} \\
D_{i}
\end{array}\right] \Lambda_{i}^{-1}\left[\begin{array}{ll}
\tilde{N} & \tilde{D}
\end{array}\right]+ \\
& {\left[\begin{array}{c}
D \\
-N
\end{array}\right] Q \Lambda_{i}^{-1}\left[\begin{array}{ll}
\tilde{N} & \tilde{D}
\end{array}\right]}
\end{aligned}
$$

so the map from $e(t)$ to $\left[u_{w}(t) y(t)\right]^{T}$ is given by $W_{1} T\left(C_{Q}, \hat{G}\right) W_{2}$. The affine relation in $Q$ for the minimization of (3) will be exploited in a (Recursive) Least Squares closed-loop data-based solution in the weighted REACT algorithm.

\section{Closed-loop data-based minimization}

A data-based solution for minimizing the combined variance of the performance signal $y(t)$ and a filtered control signal $u_{w}(t)=W(q) u(t)$ given in (2) can be formulated based on the closed-loop data obtained from the feedback system in (2). The closed-loop data-based solution can be formulated by first writing $y(t)=\hat{G} u(t)+H e(t)$ as $y(t)=\tilde{D}^{-1} \tilde{N} u(t)+$ $H e(t)$. Subsequently, the filtered noise $\Lambda_{i}^{-1} \tilde{D} H e(t)$ can be written as $\Lambda_{i}^{-1} \tilde{D} H e(t)=\Lambda_{i}^{-1} \tilde{D} y(t)-\Lambda_{i}^{-1} \tilde{N} u(t)$. Based on this analysis, we can define $w(t)=\Lambda_{i}^{-1} D H e(t)$ as a filtered closed-loop signal

$$
w(t)=\Lambda_{i}^{-1}\left[\begin{array}{ll}
\tilde{N} & \tilde{D}
\end{array}\right]\left[\begin{array}{c}
-u(t) \\
y(t)
\end{array}\right]
$$

and it should be noted that $w(t)$ is bounded, as $\Lambda_{i}^{-1} \in \mathcal{R} \mathcal{H}_{\infty}$ with $T\left(C_{i}, \hat{G}\right) \in \mathcal{R} \mathcal{H}_{\infty}$ and obviously $(\tilde{D}, \tilde{N}) \in \mathcal{R} \mathcal{H}_{\infty}$. The signal $w(t)=\Lambda_{i}^{-1} \tilde{D} H e(t)$ in (4) reconstructs the (filtered) noise signal via the closed-loop signals $u(t)$ and $y(t)$.

The affine relation in the controller perturbation $Q$ summarized in Corollary 1 allows us to write

$$
\begin{aligned}
{\left[\begin{array}{c}
\gamma u_{w}(t) \\
y(t)
\end{array}\right] } & =\left(\left[\begin{array}{c}
-\gamma W N_{i} \\
D_{i}
\end{array}\right]-\left[\begin{array}{c}
\gamma W D \\
N
\end{array}\right] Q\right) \Lambda_{i}^{-1} \tilde{D} H e(t) \\
& =\left[\begin{array}{c}
-\gamma W N_{i} \\
D_{i}
\end{array}\right] w(t)-\left[\begin{array}{c}
\gamma W D \\
N
\end{array}\right] Q w(t)
\end{aligned}
$$

By allowing a parametrization of $Q(\theta)$, the error signal

$$
\varepsilon(t, \theta)=\left[\begin{array}{c}
-\gamma N_{i} \\
D_{i}
\end{array}\right] w(t)-\left[\begin{array}{c}
\gamma D \\
N
\end{array}\right] Q(\theta) w(t)
$$

will be linear in the parameter $\theta$ if and only if $Q(\theta) \in \mathcal{R} \mathcal{H}_{\infty}$ is parameterized linearly in $\theta$. An obvious choice that ensures 
$Q(\theta) \in \mathcal{R H}_{\infty} \forall \theta$ that is parametrized linearly in $\theta$ would be a (finite dimensional) FIR model

$$
Q(q, \theta)=b_{0}+\sum_{k=0}^{\bar{k}-1} b_{k+1} q^{-k-1}, \theta=\left[\begin{array}{llll}
b_{0} & b_{1} & \cdots & b_{\bar{k}}
\end{array}\right]
$$

of order $\bar{k}$. This parameterization of $Q(q, \theta)$ allows a convex optimization of (3) over $\theta$ due to the fact that

$$
\begin{aligned}
& \| \gamma u_{w} \\
& y \|_{2}=\lim _{N \rightarrow \infty} \frac{1}{N} \sum_{t=1}^{N} \varepsilon(t, \theta)^{T} \varepsilon(t, \theta) \\
&=\left\|\left[\begin{array}{c}
-\gamma W N_{i} \\
D_{i}
\end{array}\right] w(t)-\left[\begin{array}{c}
\gamma W D \\
N
\end{array}\right] Q(\theta) w(t)\right\|_{2}
\end{aligned}
$$

using Parseval's theorem [19]. It should be noted that the parametrization of $Q(q, \theta)$ in (6) will not limit the shape and size of $\left|Q\left(e^{j \omega}, \theta\right)\right|$, possibly leading to a high frequency gain due to the FIR filter. The parametrization of $Q(q, \theta)$ can be extended with a fixed stable denominator to allow for additional filtering and limit the control to a specific frequency range. Additionally, with information on desirable pole locations for $Q(q, \theta)$ a parametrization using rational orthogonal basis function [20] can be used, as linearity and stability are preserved. In this paper we left these options open and assumed no information on desirable frequency range and/or pole location in $Q(q, \theta)$.

\section{Recursive Solutions}

To anticipate changes in the spectrum $\Phi_{v}(\omega)$, the combined variance of the performance signal $y(t)$ and a filtered control signal $u_{w}(t)$ is computed only over a finite number of time samples. The finite time computation is used to formulate a Recursive Least Squares (RLS) solution.

For a Single Input Single Output (SISO) system (with $\tilde{D}=$ $D$ and $\tilde{N}=N$ ) the expression of the error signal in (5) can be simplified to $\varepsilon(t, \theta)=y_{f}(t)-\operatorname{diag}\{Q(\theta)\} u_{f}(t)$ where $Q(\theta)$ is now a scalar FIR transfer function as in (6) and

$$
\operatorname{diag}\{Q(\theta)\}=\left[\begin{array}{cc}
Q(\theta) & 0 \\
0 & Q(\theta)
\end{array}\right]
$$

and $y_{f}(t)$ denotes the filtered output and $u_{f}(t)$ denotes the filtered input signals

$$
\begin{gathered}
y_{f}(t)=\left[\begin{array}{c}
-\gamma W N_{i} \\
D_{i}
\end{array}\right] w(t), u_{f}(t)=\left[\begin{array}{c}
\gamma W D \\
N
\end{array}\right] w(t) \\
w(t)=\frac{D}{\Lambda_{i}} y(t)-\frac{N}{\Lambda_{i}} u(t)
\end{gathered}
$$

To formulate a recursive solution for the minimization

$$
\begin{gathered}
\hat{\theta}_{t}=\min _{\theta} \frac{1}{t} \sum_{\tau=0}^{t} \varepsilon(\tau, \theta)^{T} \varepsilon(\tau, \theta), \\
\varepsilon(t, \theta)=y_{f}(t)-\operatorname{diag}\{Q(\theta)\} u_{f}(t)
\end{gathered}
$$

for a linearly parametrized scalar $Q(q, \theta)$, the error $\varepsilon(t, \theta)$ can be written into a linear regression form

$$
\begin{aligned}
\varepsilon(t, \theta) & =y_{f}(t)-\phi(t)^{T} \theta \in \mathcal{R}_{2 \times 1}, \text { with } \\
\phi(t)^{T} & =\left[\begin{array}{llll}
u_{f}(t) & u_{f}(t-1) & \cdots & u_{f}(t-\bar{k})
\end{array}\right] \in \mathcal{R}_{2 \times \bar{k}+1} \\
\theta & =\left[\begin{array}{llll}
b_{0} & b_{1} & \cdots & b_{\bar{k}}
\end{array}\right]^{T} \in \mathcal{R}_{\bar{k}+1 \times 1}
\end{aligned}
$$

where the regressor $\phi(t)$ contains past and/or filtered versions of the input signal $u_{f}(t)$.

A standard RLS update algorithm [19], [11] can be summarized by the three iterative steps of an a posteriori prediction error update:

$$
\varepsilon\left(t, \hat{\theta}_{t-1}\right)=y(t)-\phi(t)^{T} \hat{\theta}_{t-1}
$$

a time weighted covariance update:

$$
\begin{aligned}
P_{t}= & P_{t-1}- \\
& P_{t-1} \phi(t)\left[\phi(t)^{T} P_{t-1} \phi(t)+I_{2 \times 2}\right]^{-1} \phi(t)^{T} P_{t-1}
\end{aligned}
$$

a parameter update:

$$
\hat{\theta}_{t}=\hat{\theta}_{t-1}+P(t) \phi(t) \varepsilon\left(t, \hat{\theta}_{t-1}\right)
$$

and initialized at $t=1$ by $\theta_{0}=0$ and $P_{0}=\mu I, \mu>>1$. Typically, one chooses $\mu$ to be a large number to allow for fast and aggressive initial parameter updates [19].

Unfortunately, the standard RLS formulation as given above suffers from two main drawback: (a) Choosing a large value of $\mu>>1$ to allow for fast initial parameter updates $\theta_{t}$ causes a large volatility in $\theta_{t}-\theta_{t-1}$ during the initial parameter updates and (b) for $\mu>>1, \lim _{t \rightarrow \infty} P_{t}=0$, causing the parameter updates to converge to a stationary point $\lim _{t \rightarrow \infty} \theta_{t}=\theta^{\star}$. Item (a) is problematic, since the parameter $\theta_{t}$ is used directly in the controller perturbation $Q\left(q, \theta_{t}\right)$ and causes large volatility in the controller updates $C_{Q}$ and the resulting control signal $u(t)=-C_{Q} y(t)$, possibly saturating or destabilizing the time varying control system.

To address drawback (a), the controller perturbation $Q\left(q, \tilde{\theta}_{t}\right)$ is updated with a parameter $\tilde{\theta}_{t}$ that is a time-filtered version of the estimated parameter $\hat{\theta}_{t}$ according to

$$
\tilde{\theta}_{t}=(1-\delta) \hat{\theta}_{t}+\delta \tilde{\theta}_{t-1}
$$

where $0 \leq \delta<1$. The closer $\delta$ is to 1 , the more filtering and the slower the parameter $\tilde{\theta}_{t}$ is adjusted due to step-wise changes in $\hat{\theta}_{t}$.

To address drawback (b), a regularization is added to the time weighted covariance update $P_{t}$ in (11) according to

$$
\begin{aligned}
P_{t}= & P_{t-1}+\lambda_{0} I- \\
& P_{t-1} \phi(t)\left[\phi(t)^{T} P_{t-1} \phi(t)+I_{2 \times 2}\right]^{-1} \phi(t)^{T} P_{t-1}
\end{aligned}
$$

where $\lambda_{0}>0$. The regularization has a close relation to Kalman filtering updates when parameters are time varying [19] and ensure $P_{t}>0$, even as $t \rightarrow \infty$. This means that the parameter estimate $\theta_{t}$ will not converge to a stationary point, and is able to change at any time $t$ in case there is a perturbation in the spectrum $\Phi_{v}(\omega)=\left|H\left(e^{j \omega}\right)\right|^{2} \nu$ of the noise disturbance $v(t)=H e(t)$.

\section{ApPlicAtion to VibRATION BENCHMARK}

\section{A. Summary of Algorithm}

The mechanical test bed of the benchmark on adaptive regulation [24], [25] is used to verify the proposed weighted REACT algorithm for adaptive regulation of disturbances. The benchmark problem requires adaptive regulation of 
multiple unknown and time-varying harmonic disturbances in the range between 50 and $100 \mathrm{~Hz}$ at different levels of complexity. Disturbances enter the mechanical system via a Primary Path (PP) actuator, while feedback control can be done via a Secondary Path (SP) actuator. The levels of complexity are distinguished by the number of harmonic disturbances that may be present at any given time.

Only a model $\hat{G}$ of the SP actuator is used in the weighted REACT algorithm, e.g. the PP actuator is assumed to be unknown and part of the unknown disturbance dynamics. Using experimental data, a model $\hat{G}$ of the SP actuator is created based on frequency domain curve fitting and used to design an initial controller $C_{i}$ via a standard $\mathcal{H}_{2}$ control design that minimizes (3) in the presence of a white noise disturbance signal $v(t)$. The resulting model $\hat{G}$ accurately models the resonance modes in SP over a frequency range up to $150 \mathrm{~Hz}$ and reveals that the SP actuator has a nearly undamped zero located at $98 \mathrm{~Hz}$.

To ensure the controller $C_{i}$ is robust against unmodelled dynamics above $150 \mathrm{~Hz}$ in $\hat{G}$, the monic filter $W$ in (3) is chosen as

$$
W(q)=\frac{q^{4}-1.57 q^{3}+1.276 q^{2}-0.4844 q+0.0762}{q^{4}+2.8 q^{3}+2.94 q^{2}+1.372 q+0.2401}
$$

representing a 4th order high-pass filter with a cut-off frequency at $150 \mathrm{~Hz}$, a DC-gain of $-30 \mathrm{~dB}$ and a gain of $50 \mathrm{~dB}$ at the Nyquist frequency of $400 \mathrm{~Hz}$. To further limit the control signal during adaptation, the value of $\gamma$ is set to $\gamma=1.4$ and the choices for $W$ and $\gamma$ will be kept the same during all adaptive regulation experiments of $C_{Q}$.

The controller perturbation $Q(\theta)$ is parameterized as (6) with $\bar{k}=29$. Choosing $\bar{k}$ larger allows more freedom in adapting the controller $C_{Q}$, especially for multiple harmonic disturbances at higher level of complexity of the benchmark. For the real-time implementation sampled at $800 \mathrm{~Hz}$, the size of $\bar{k}=29$ was limited by the available computational power to implement the recursive estimation of the parameter $\hat{\theta}_{t}$ via the three computational steps outlines in (10), (14) and (12). The filtered signals $u_{f}$ and $y_{f}$ in (8) are obtained by choosing $(N, D)$ and $\left(N_{i}, D_{i}\right)$ as normalized coprime factorizations of respectively $\hat{G}$ and $C_{i}$.

To avoid effects of initial parameter conditions during adaptation, the controller $C_{Q}\left(\tilde{\theta}_{t}\right)$ is updated with the filtered parameter $\tilde{\theta}_{t}$ in (13). For the recursive adaptation with the weighted REACT, a fixed initialization is chosen with $\hat{\theta}_{t}=0$ and $P_{0}=\mu I, \mu=10^{4}$ and $\delta=0.995$ in (13) and $\lambda_{0}=2$ in (14) at $800 \mathrm{~Hz}$ sampling.

\section{B. Results for level 2 frequency disturbance}

For the initial evaluation of performance and transient effects, harmonic disturbance with two distinct frequencies in the range $50-95 \mathrm{~Hz}$ are applied at an unknown time, requiring the controller $C_{Q}$ to regulate itself to minimize the criteria in (3). For evaluation of the weighted REACT algorithm both a Simple Step change, Multiple Step changes and Chirp changes in the frequencies of the harmonic disturbances are administered. To illustrate the effectiveness of the weighted
REACT algorithm, a typical response for a level 2 frequency disturbance is depicted in Fig. 1.
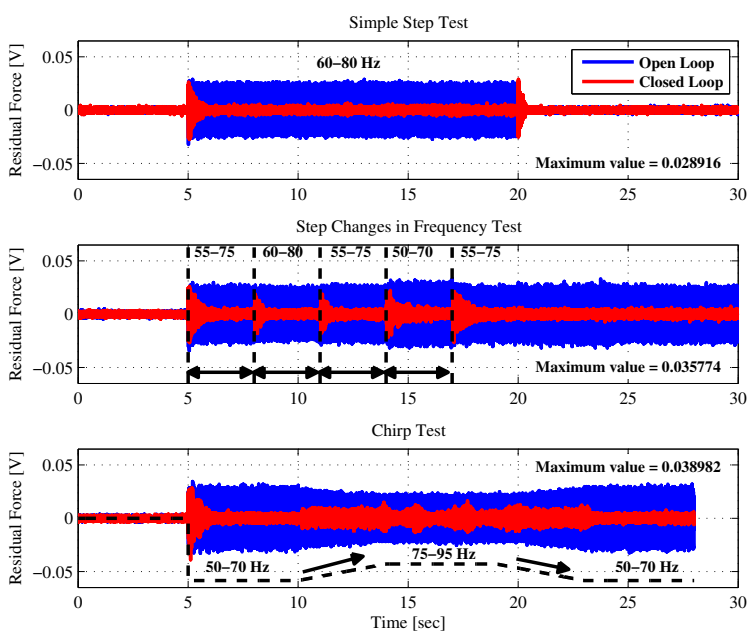

Fig. 1. Experimental results of open loop (blue) and closed-loop (red) output time sequences for a Simple Step change, Multiple Step changes and Chirp change in the frequencies of the level 2 harmonic disturbance. The 2 distinct frequencies of the harmonic disturbances and the maximum values of the output signal in closed-loop are indicated in the figure.

The adaptive regulation results with the weighted REACT for other level 2 harmonic disturbance in the frequency range of $50-95 \mathrm{~Hz}$ look very similar to Fig. 1 with an excellent suppression of the sinusoidal disturbance down to the noise level of the experiment. This is also confirmed by the spectral analysis of the controlled output signal in the Simple Step test depicted in Fig. 2. that reveals excellent disturbance rejection of the 60 and $80 \mathrm{~Hz}$ harmonics, but also shows an amplification in neighboring frequencies. It also shows that the choice of the high pass filter $W(q)$ in (15) limits the control energy with no observable amplification of the PSD above $150 \mathrm{~Hz}$. As noted before, additional modifications of the filter $W(q)$ or extending the parametrization of $Q(q, \theta)$ with a fixed stable denominator will allow for additional limitations of the control to a specific frequency range.

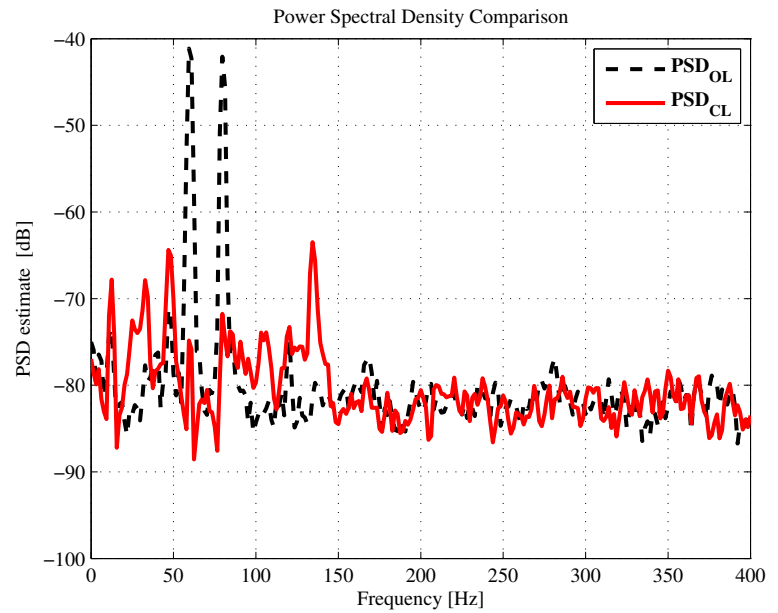

Fig. 2. Experimental results of the open loop (dashed black line) and closed-loop (red line) Power Spectral Density (PSD) estimates of the output in case of a Simple Step change of the level 2 harmonic disturbance. 


\begin{tabular}{|c|c|c|c|c|c|c|c|}
\hline $\begin{array}{c}\text { Freq. } \\
{[\mathrm{Hz}-\mathrm{Hz}]}\end{array}$ & $\begin{array}{c}G_{A} \\
{[\mathrm{~dB}]}\end{array}$ & $\begin{array}{c}D_{A} \\
{[\mathrm{~dB}-\mathrm{dB}]}\end{array}$ & $\begin{array}{c}M_{A} \\
{[\mathrm{~dB} @ \mathrm{~Hz}]}\end{array}$ & $\begin{array}{c}T_{N} \\
{\left[10^{-3} \mathrm{~V}^{2}\right]}\end{array}$ & $\begin{array}{c}R_{N} \\
{\left[10^{-3} \mathrm{~V}^{2}\right]}\end{array}$ & $\begin{array}{c}T_{M} \\
{[\mathrm{mV}]}\end{array}$ & $\begin{array}{c}T_{t} \\
{[\mathrm{msec}]}\end{array}$ \\
\hline $50-70$ & 36.96 & $36.41-31.44$ & $11.05 @ 90.63$ & 69.99 & 8.04 & 36.03 & 615.00 \\
\hline $55-75$ & 34.79 & $35.46-30.59$ & $16.29 @ 132.80$ & 47.19 & 7.97 & 30.14 & 431.25 \\
\hline $60-80$ & 33.73 & $33.69-29.69$ & $17.83 @ 134.40$ & 41.83 & 8.00 & 27.46 & 413.75 \\
\hline $65-85$ & 28.29 & $29.04-26.64$ & $14.61 @ 132.80$ & 61.29 & 13.20 & 31.17 & 490.00 \\
\hline $70-90$ & 27.13 & $30.13-24.30$ & $21.29 @ 103.10$ & 42.02 & 13.52 & 34.83 & 250.00 \\
\hline $75-95$ & 23.09 & $29.71-19.80$ & $24.59 @ 132.80$ & 51.50 & 19.33 & 36.06 & 9515.00 \\
\hline
\end{tabular}

TABLE I

EXPERIMENTAL RESULTS OF THE PERFORMANCE AND TRANSIENT PARAMETERS FOR LEVEL 2 SINGLE STEP CHANGE IN HARMONIC DISTURBANCE.

\begin{tabular}{|c|c|c|c|c|}
\hline S\# & $\begin{array}{c}\text { Freq. } \\
{[\mathrm{Hz}]}\end{array}$ & $\begin{array}{c}T_{N} \\
{\left[10^{-3} \mathrm{~V}^{2}\right]}\end{array}$ & $\begin{array}{c}T_{M} \\
{[\mathrm{mV}]}\end{array}$ & $\begin{array}{c}T_{t} \\
{[\mathrm{msec}]}\end{array}$ \\
\hline \multirow{4}{*}{$\approx$} & $55,75 \rightarrow 60,80$ & 19.10 & 18.62 & 208.75 \\
\cline { 2 - 5 } & $60,80 \rightarrow 55,75$ & 21.55 & 18.62 & 160.00 \\
\cline { 2 - 5 } & $55,75 \rightarrow 50,70$ & 42.09 & 22.29 & 197.50 \\
\cline { 2 - 5 } & $50,70 \rightarrow 55,75$ & 47.26 & 26.73 & 102.50 \\
\hline \multirow{4}{*}{$\approx$} & $70,90 \rightarrow 75,95$ & 25.76 & 13.70 & 198.75 \\
\cline { 2 - 5 }$\approx$ & $75,95 \rightarrow 70,90$ & 34.91 & 20.62 & 151.25 \\
\cline { 2 - 5 } & $70,90 \rightarrow 65,85$ & 23.62 & 18.60 & 107.50 \\
\cline { 2 - 5 } & $65,85 \rightarrow 70,90$ & 33.15 & 18.60 & 96.25 \\
\hline
\end{tabular}

TABLE II

EXPERIMENTAL RESULTS OF THE TRANSIENT PARAMETERS FOR LEVEL 2 Multiple Step Changes in harmonic Disturbance.

The performance and transient effects for the different level 2 harmonic disturbances are summarized in Table I and Table II. With $L_{e}=16000$ as the last sample when the disturbance is present in case of a Single Step test, the performance is measured via the global attenuation

$$
G_{A}=20 \log ^{10} \frac{\sum_{t=4800-L_{e}}^{L_{e}} y_{o l}(t)^{2}}{\sum_{t=4800-L_{e}}^{L_{e}} y_{c l}(t)^{2}}
$$

and the specific Disturbance Attenuation $D_{A}$ by inspecting the frequency domain plot (Fig. 2) at the frequencies of the harmonic disturbance. Part of the performance evaluation is also the maximum amplification $M_{A}$ of any other (neighboring) frequencies and the residual norm $R_{N}$ in Table I. With $L_{i}$ as the first sample when the harmonic disturbance is applied, transient effects are characterized by inspecting the transient norm $T_{N}=\sum_{t=L_{i}}^{L_{i}+2400} y_{c l}(t)^{2}$, the maximum value $T_{M}$ during transient and the transient time $T_{t}$ in Table I and Table II. The results in the tables indicate that for a level 2 harmonic disturbance on average a $30 \mathrm{~dB}$ reduction of the disturbance is obtained, except for the $95 \mathrm{~Hz}$ harmonic disturbance that is close to the undamped zero at $98 \mathrm{~Hz}$ in the SP actuator. During ramp up and down for the level 2 chirp experiments the Mean Square Error (MSE) is respectively $1.56 \cdot 10^{-5} \mathrm{~V}^{2}$ and $1.51 \cdot 10^{-5} \mathrm{~V}^{2}$, whereas the maximum value of the output was respectively $14.9 \mathrm{mV}$ and $12.5 \mathrm{mV}$.

\section{Results for level 3 frequency disturbance}

For the final evaluation of performance and transient effects, harmonic disturbance with three distinct frequencies in the range $50-95 \mathrm{~Hz}$ are applied at an unknown time. A typical response for such a level 3 frequency disturbance is depicted in Fig. 3 for a Simple Step change, Multiple Step changes and Chirp changes in the frequencies of the harmonic disturbances.

The spectral analysis of the controlled output signal in the Simple Step test depicted in Fig. 4 shows partial disturbance
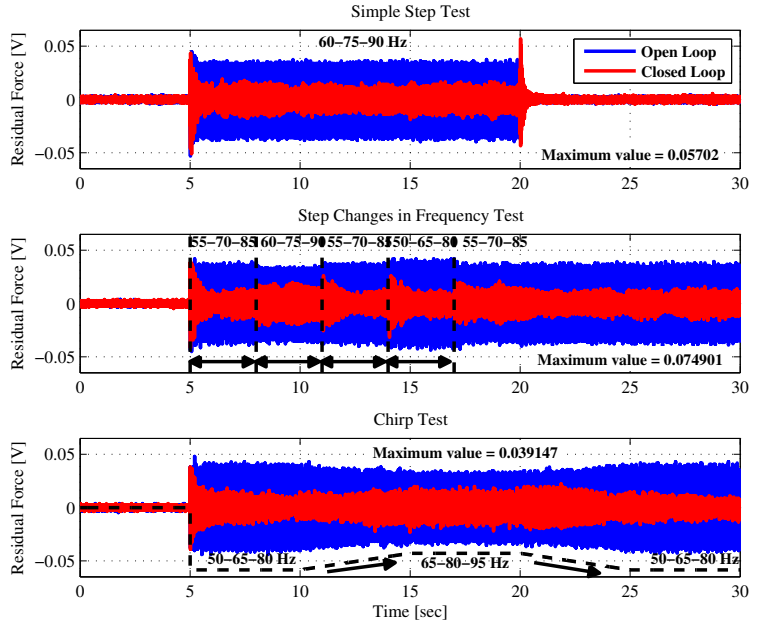

Fig. 3. Experimental results of open loop (blue) and closed-loop (red) output time sequences for a Simple Step change, Multiple Step changes and Chirp change in the frequencies of the level 3 harmonic disturbance. The 3 distinct frequencies of the harmonic disturbances and the maximum values of the output signal in closed-loop are indicated in the figure.

rejection of the 3 harmonic disturbances, with a similar amplification in neighboring frequencies. The partial disturbance rejection is caused by the weighting of the control energy during the adaptation, trading off the amount of control energy available for disturbance rejection.

For comparison purposes, the performance and transient effects for the different level 3 harmonic disturbances are summarized in Table III and Table IV. From the data given in these tables it can be observed that transient times are longer for the Single Step change test and the adaptation algorithm is selective in reducing disturbances due to the trade-off in output and control signal variance. The transient times for the Multiple Step change are reduced to a few samples due to the limited disturbance reduction obtained. During ramp up and down for the level 3 chirp experiments the Mean Square Error (MSE) is respectively $4.41 \cdot 10^{-5} \mathrm{~V}^{2}$ and $7.08 \cdot 10^{-5} \mathrm{~V}^{2}$, whereas the maximum value of the output was respectively $23.2 \mathrm{mV}$ and $22.1 \mathrm{mV}$. In all cases the robust estimation algorithm is kept the same and stability of feedback loop is maintained during adaptive regulation.

\section{Conclusions}

The weighted REACT uses a coprime factorization of a model and an initial controller to create filtered signals from closed-loop measurements for a direct estimation of a feedback controller transfer function parametrized in a YoulaKucera parametrization. A weighting on the control signal 


\begin{tabular}{|c|c|c|c|c|c|c|c|}
\hline $\begin{array}{c}\text { Freq. } \\
{[\mathrm{Hz}-\mathrm{Hz}]}\end{array}$ & $\begin{array}{c}G_{A} \\
{[\mathrm{~dB}]}\end{array}$ & $\begin{array}{c}D_{A} \\
{[\mathrm{~dB}-\mathrm{dB}]}\end{array}$ & $\begin{array}{c}M_{A} \\
{[\mathrm{~dB} @ \mathrm{~Hz}]}\end{array}$ & $\begin{array}{c}T_{N} \\
{\left[10^{-3} \mathrm{~V}^{2}\right]}\end{array}$ & $\begin{array}{c}R_{N} \\
{\left[10^{-3} \mathrm{~V}^{2}\right]}\end{array}$ & $\begin{array}{c}T_{M} \\
{[\mathrm{mV}]}\end{array}$ & $\begin{array}{c}T_{t} \\
{[\mathrm{msec}]}\end{array}$ \\
\hline $50-65-80$ & 24.02 & $27.19-7.84-24.53$ & $21.93 @ 103.10$ & 160.26 & 47.74 & 40.99 & 337.50 \\
\hline $55-70-85$ & 21.64 & $24.90-7.71-19.79$ & $20.99 @ 103.10$ & 119.40 & 52.02 & 43.46 & 270.00 \\
\hline $60-75-90$ & 13.76 & $25.91-3.14-19.96$ & $22.76 @ 132.80$ & 164.44 & 115.66 & 50.82 & 433.75 \\
\hline $65-80-95$ & 15.70 & $23.48-12.11-4.68$ & $24.34 @ 137.50$ & 150.17 & 82.79 & 53.28 & 5406.30 \\
\hline
\end{tabular}

TABLE III

EXPERIMENTAL RESULTS OF THE PERFORMANCE AND TRANSIENT PARAMETERS FOR LEVEL 3 SINGLE STEP CHANGE IN HARMONIC DISTURBANCE.

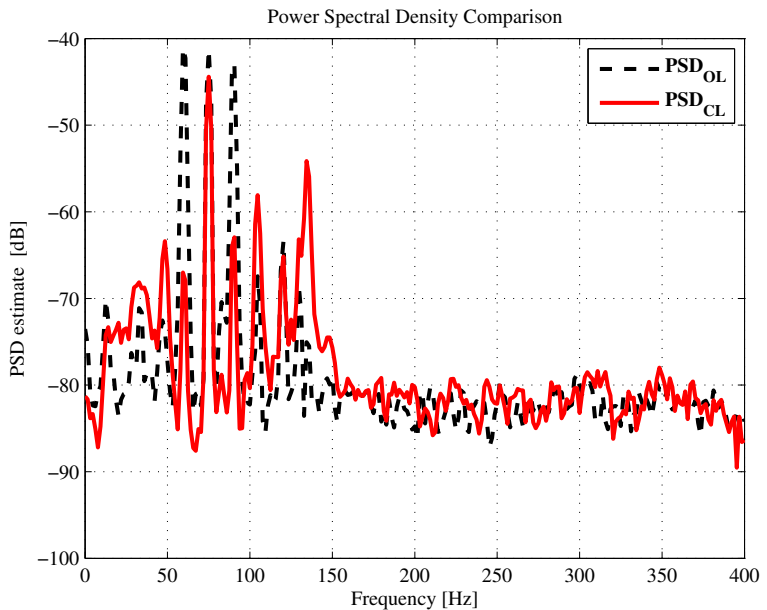

Fig. 4. Experimental results of the open loop (dashed black line) and closed-loop (red line) Power Spectral Density (PSD) estimates of the output in case of a Simple Step change of the level 3 harmonic disturbance.

\begin{tabular}{|c|c|c|c|c|}
\hline S\# & $\begin{array}{c}\text { Freq. } \\
{[\mathrm{Hz}]}\end{array}$ & $\begin{array}{c}T_{N} \\
{\left[10^{-3} \mathrm{~V}^{2}\right]}\end{array}$ & $\begin{array}{c}T_{M} \\
{[\mathrm{mV}]}\end{array}$ & $\begin{array}{c}T_{t} \\
{[\mathrm{msec}]}\end{array}$ \\
\hline \multirow{5}{*}{$\approx$} & $55,70,85 \rightarrow 60,75,90$ & 193.52 & 20.69 & 2.50 \\
\cline { 2 - 5 } & $60,75,90 \rightarrow 55,70,85$ & 109.16 & 25.88 & 2.50 \\
\cline { 2 - 5 } & $55,70,85 \rightarrow 50,65,80$ & 102.84 & 31.72 & 2.50 \\
\cline { 2 - 5 } & $50,65,80 \rightarrow 55,70,85$ & 162.39 & 20.98 & 2.50 \\
\hline \multirow{4}{*}{$\approx$} & $60,75,90 \rightarrow 65,80,95$ & 149.66 & 25.61 & 2.50 \\
\cline { 2 - 5 } & $65,80,95 \rightarrow 60,75,90$ & 229.21 & 25.61 & 2.50 \\
\cline { 2 - 5 } & $60,75,90 \rightarrow 55,70,85$ & 110.92 & 27.09 & 2.50 \\
\cline { 2 - 5 } & $55,70,85 \rightarrow 60,75,90$ & 183.66 & 23.16 & 2.50 \\
\hline
\end{tabular}

TABLE IV

EXPERIMENTAL RESULTS OF THE TRANSIENT PARAMETERS FOR LEVEL

3 Multiple Step CHANGES IN HARMONiC DisturbanCE.

is used to account for uncertainty on the plant dynamics to limit the control. The filtered signals are used to formulate a Recursive Least Squares algorithm to simultaneously minimize the variance of a performance signal and the frequency weighted control signal. Regularization and filtering of the recursive parameter updates smoothen the volatility when the adaptive regulation controller is initialized and/or adjusted to reduce periodic disturbances with sudden jumps in the frequency content. The approach is successfully demonstrated on a vibration control benchmark with excellent results on performance and transient properties.

\section{REFERENCES}

[1] B. Francis and W. Wonham, "The internal model principle of control theory," Automatica, vol. 12, no. 5, pp. 457-465, September 1976.

[2] M. Tomizuka, T. C. Tsao, and K. Chew, "Analysis and synthesis of discrete-time repetitive controllers," ASME Journal of Dynamic Systems, Measurement, and Control, vol. 111, no. 3, pp. 353-358, September 1989.

[3] M. Steinbuch, "Repetitive control for systems with uncertain periodtime," Automatica, vol. 38, no. 12, pp. 2103-2109, 2002.
[4] M. Bodson, "Rejection of periodic disturbances of unknown and timevarying frequency," International Journal of Adaptive Control and Signal Processing, vol. 19, no. 2-3, pp. 67-88, March-April 2005.

[5] G. Pipeleers, J. Swevers, and B. Demeulenaere, Optimal Linear Controller Design for Periodic Inputs. Berlin, Heidelberg, Germany: Springer Verlag, Lectures Notes in Control and Informations Sciences, M. Thoma, F. Allgöwer and M. Morari (Eds.), 2009.

[6] K. Zhou and J. Doyle, Essentials of Robust Control. Upper Saddle River, NJ: Prentice Hall, 1997.

[7] M. Seron, J. Braslavsky, P. Kokotovic, and D. Mayne, "Feedback limitations in nonlinear systems: From bode integrals to cheap control," IEEE Trans. on Automatic Control, vol. 44, pp. 829 - 833, 1999.

[8] D. Ortiz, J. S. Freudenberg, and R. H. Middleton, "Feedback limitations of linear sampled-data periodic digital control," Int. Journal of Robust and Nonlinear Control, vol. 10, pp. 729-745, 2000.

[9] C. Du and L. Xie, Modeling and Control of Vibration in Mechanical Systems. CRC Press, 2010.

[10] R. de Callafon and J. Zeng, "Recursive filter estimation for feedforward noise cancellation with acoustic coupling," Journal of Sound and Vibration, vol. 291, pp. 1061-1079, 2006.

[11] I. Landau, A. Constantinescu, and M. Alma, "Adaptive regulation - rejection of unknown multiple narrow band disturbances," in Proc. 17th Mediterranean Conference on Control and Automation, Thessaloniki, Greece, 2009, pp. 1056-1065.

[12] M. Bodson, "Rejection of periodic disturbances of unknown and timevarying frequency," International Journal of Adaptive Control and Signal Processing, vol. 19, no. 2-3, pp. 67-88, March-April 2005.

[13] R. Longman, "Iterative learning control and repetitive control for engineering practice," Int. Journal of Control, vol. 73, pp. 930-954, 2000.

[14] Y.-Q. Chen, K. Moore, J. Yu, and T. Zhang, "Iterative learning control and repetitive control in hard disk drive industry - a tutorial." Int. J. Adapt. Control and Signal Processing, vol. 22, pp. 325-343, 2007.

[15] M. Tomizuka, "Dealing with periodic disturbances in controls of mechanical systems," Annual Reviews in Control, vol. 32, pp. 193199, 2008.

[16] I. Landau, R. Lozano, M. M'Saad, and A. Karimi, "Adaptive regulation - rejection of unknown disturbances," Communication and Control Engineering, pp. 477-497, 2011.

[17] C. Kinney, H. Fang, R. de Callafon, and M. Alma, "Robust estimation and automatic controller tuning in vibration control of time varying harmonic disturbances," in Prepr. 18th IFAC World Congress, I. Milan, Ed., 2011, pp. 5401-5406.

[18] B. Anderson, "From Youla-Kucera to identification, adaptive and nonlinear control," Automatica, vol. 34, pp. 1485-1506, 1998.

[19] L. Ljung, System Identification-Theory for the User, 2nd ed. Englewood Cliffs, NJ USA: Prentice-Hall, 1999.

[20] P. Heuberger, P. Van den Hof, and B. Wahlberg, Modelling and Identification with Rational Orthogonal Basis Functions. SpringerVerlag, London, 2005.

[21] H. Niemann, "Dual Youla parameterisation," IEE Proc. Control Theory Appl., vol. 150, pp. 493-497, 2003.

[22] S. Douma, P. Van den Hof, and O. Bosgra, "Controller tuning freedom under plant identification uncertainty: Double youla beats gap in robust stability," Automatica, vol. 39, pp. 325-333, 2003.

[23] S. Boyd and L. Vandenberghe, Convex Optimization. Cambridge University Press, March 2004.

[24] I. Landau, T. Airimitoaie, A. Castellanos Silva, and G. Buche, "Benchmark on adaptive regulation," http://www.gipsa-lab.grenoble-inp.fr/ ioandore. landau/benchmark_adaptive_regulation/.

[25] —, "Benchmark on adaptive regulation: Rejection of unknown/timevarying multiple narrow band disturbances," in Proc. 12th European Control Conference, Zürich, Zwitserland, 2013. 